\title{
Abortos, malformações congênitas e falhas reprodutivas espontâneas em caprinos causados na intoxicação pelas folhas da catingueira, Poincianella pyramidalis (sin. Caesalpinia pyramidalis) ${ }^{1}$
}

\author{
Maria de Fátima Souza ${ }^{2}$, Isadora T.F. Bezerra ${ }^{3}$, Francisca M.S. Barbosa ${ }^{3}$, \\ Valdemar C. Rocha ${ }^{3}$, Monica S. Sousa ${ }^{3}$, Temístocles S. Oliveira Neto ${ }^{3}$, \\ Patrícia B. Lacerda-Lucena ${ }^{4}$ e Ricardo B. Lucena ${ }^{2,3 *}$
}

\begin{abstract}
Souza M.F., Bezerra I.T.F., Barbosa F.M.S., Rocha V.C., Sousa M.S., Oliveira Neto T.S., Lacerda-Lucena P.B. \& Lucena R.B. 2018. [Spontaneous abortion, congenital malformation and reproductive losses in goats due to poisoning by the leaves of Poincianella pyramidalis (syn. Caesalpinia pyramidalis).] Abortos, malformações congênitas e falhas reprodutivas espontâneas em caprinos causados pela intoxicação pelas folhas da catingueira, Poincianella pyramidalis (sin. Caesalpinia pyramidalis). Pesquisa Veterinária Brasileira 38(6):1051-1057. Laboratório de Patologia Veterinária, Departamento de Ciências Veterinárias, Centro de Ciências Agrárias, Universidade Federal da Paraíba, Campus II, Areia, PB 58397-000, Brazil. E-mail: lucena.rb@gmail.com

The aim of this study was to investigate the effects of the leaves of the tree Poincianella pyramidalis (Tul.) L.P. Queiroz as a spontaneous cause of abortion, malformation and reproductive failure in goat herds in the Brazilian semiarid region. Epidemiological investigations, clinical evaluation of goats affected by malformation and necropsy of aborted fetuses was carried out on 12 farms located in the municipalities of Gurjão and Soledade, state of Paraíba, and on a farm located at the municipality of Pau dos Ferros, state of Rio Grande do Norte. The study considered only the farms where occurred predominantly $P$. pyramidalis among teratogenic plants. On the farm located in the municipality of Pau dos Ferros the occurrence of frequent abortion and birth of malformed goats, affecting $90 \%$ of pregnant goats, was observed. On the 12 farms belonging to the municipalities of Gurjão and Soledade, spontaneous cases of embryonic mortality, abortion and malformation occurred in 257 goats ( $41.1 \%$ of goats). Arthrogryposis was the main congenital malformation observed. Necropsy and histopathological exams of four fetuses and their placentas did not detect the presence of infectious agents. The cases of embryonic mortality, abortion and malformation were predominantly concentrated during the seasons of the year when pasture was scarce, but $P$. pyramidalis was available, due to the occurrence of pre-seasonal rains, which did not allow the pasture to germinate but $P$. pyramidalis to regrowth. The findings of this study confirm that this plant significantly interferes in the reproductive efficiency of semi-arid goat herds.
\end{abstract}

INDEX TERMS: Poisonous plants, abortion, malformation, reproductive losses, goats, catingueira, Poincianella pyramidalis, Caesalpinia pyramidalis, plant poisoning, teratogenic plant, arthrogryposis, semiarid, toxicoses.

\footnotetext{
${ }^{1}$ Recebido em 6 de junho de 2017.

Aceito para publicação em 21 de junho de 2017.

Parte da Dissertação de Mestrado do primeiro autor.

${ }^{2}$ Programa de Pós-Graduação em Ciência Animal, Centro de Ciências Agrárias (CCA), Universidade Federal da Paraíba (UFPB), Campus II, Areia, PB 58397-000, Brasil. E-mail: fatsou_capri@yahoo.com.br

${ }^{3}$ Laboratório de Patologia Veterinária, Hospital Veterinário, Departamento de Ciências Veterinárias, Centro de Ciências Agrárias (CCA), Universidade
}

Federal da Paraíba (UFPB), Campus II, Areia, PB 58397-000. E-mail: isadora.f.ilgueira@hotmail.com, franciscaaraguaia@gmail.com, valdemar_ cavalcante@hotmail.com, temivet@hotmail.com; *Autor para correspondência: lucena.rb@gmail.com

${ }^{4}$ Programa de Doutorado Integrado em Zootecnia, Centro de Ciências Agrárias (CCA), Universidade Federal da Paraíba (UFPB), Campus II, Areia, PB 58397-000. E-mail: patriciabarbosadelacerda@gmail.com 
RESUMO.- 0 objetivo deste estudo foi investigar os efeitos de folhas da espécie arbórea Poincianella pyramidalis (Tul.) L.P. Queiroz como causa espontânea de abortos, malformações e falhas reprodutivas em rebanhos caprinos no semiárido brasileiro. Foram realizadas investigações epidemiológicas, avaliações clínicas de caprinos acometidos por malformações e necropsias de fetos abortados em 12 propriedades localizadas nos municípios de Gurjão e Soledade, na Paraíba, e no município de Pau dos Ferros, Rio Grande do Norte. 0 estudo considerou apenas as propriedades que tinham basicamente a presença de $P$. pyramidalis entre as plantas teratogênicas. Na propriedade localizada no município de Pau dos Ferros foi verificada a ocorrência de grande número de abortos e o nascimento de cabritos malformados, acometendo $90 \%$ das cabras gestantes. Nas 12 propriedades pertencentes aos municípios de Gurjão e Soledade na Paraíba os casos espontâneos de mortalidade embrionária, abortos e malformações acometeram 257 cabras (41,1\% das cabras dos rebanhos). A artrogripose foi a principal malformação congênita observada. Exames de necropsia e histopatológico de quatro fetos acompanhados das placentas não constataram a presença de agentes infecciosos. Os casos de perda embrionária, abortos e malformações foram concentrados predominantemente nas épocas do ano em que a pastagem estava escassa, mas havia disponibilidade de $P$. pyramidalis nas propriedades, devido à ocorrência das chuvas da pré-estação, que não permitiu a germinação de pasto, mas a rebrotação de P. pyramidalis. Os achados desse estudo comprovam que esta planta interfere consideravelmente na eficiência reprodutiva dos rebanhos caprinos do Semiárido.

TERMOS DE INDEXAÇÃO: Plantas tóxicas, abortos, malformações congênitas, falhas reprodutivas, caprinos, intoxicação por plantas, Poincianella pyramidalis, Caesalpinia pyramidalis, caprinos, intoxicação por plantas, planta teratogênica, artrogripose, semiárido, toxicoses.

\section{INTRODUÇÃO}

Anomalias congênitas causam perdas embrionárias e abortos que afetam a eficiência reprodutiva dos rebanhos caprinos do Semiárido (Dantas et al. 2012), resultando em sérias perdas econômicas para os criadores. Dentre essas anomalias, as malformações representam 7,62\% das causas de mortes neonatais de cabritos no Nordeste brasileiro (Medeiros et al. 2005). Nesta Região, os rebanhos caprinos criados extensivamente com livre acesso às plantas tóxicas (Dantas et al. 2010), são acometidos por vários tipos de malformações congênitas, tais como as anomalias ósseas craniofaciais, malformações oculares e artrogripose (Medeiros et al. 2005).

Plantas tóxicas de interesse pecuário causam prejuízos a produtores em todo o mundo. No Brasil, algumas plantas causam sérias perdas econômicas devido à indução de abortos, malformações e infertilidade (Riet-Correa \& Medeiros 2001, Riet-Correa et al. 2007). No nordeste do Brasil, a planta Mimosa tenuiflora (conhecida como "jurema preta") foi comprovada como teratogênica por induzir abortos e malformações em caprinos e ovinos (Pimentel et al. 2007, Dantas et al. 2012), e a planta Aspidosperma pyrifolium (conhecida como "pereiro") como importante causa de abortos em caprinos (Medeiros et al. 2004). Casos de malformações e abortos têm sido relatos por criadores em propriedades com rara ocorrência ou ausência de Mimosa tenuiflora e Aspidosperma pyrifolium. Porém, nestas propriedades geralmente há grande quantidade da árvore
Poincianella pyramidalis (Tul.), conhecida popularmente como "catingueira", apontada por alguns criadores como planta teratogênica.

Poincianella pyramidalis é uma árvore endêmica da região Nordeste do Brasil (Agra et al. 2007, Albuquerque et al. 2007). Na medicina popular, a entrecasca dessa planta é comumente usada para o tratamento das inflamações e possui ação diurética, dispéptica, digestiva, antipirética e expectorante (Silva \& Matos 1998, Mendes et al. 2000, Agra et al. 2007, Bahia et al. 2010). Porém, um estudo realizado na Bahia comprovou que a "catingueira" causa falhas reprodutivas e malformações em pequenos ruminantes (Reis et al. 2016). Outros estudos relatam casos de toxicidade em moluscos e abelhas, indicando a presença de componentes tóxicos na planta (Luna et al. 2005, Melo et al. 2013).

Os problemas reprodutivos em caprinos tem se intensificado nos últimos anos em diferentes municípios do semiárido nordestino em decorrência do grave quadro de seca, caracterizada por baixa disponibilidade de pastagens. Por outro lado, nessas propriedades há predominância de plantas tóxicas que são mais resistentes à estiagem, como $P$. pyramidalis. Assim, este trabalho objetivou descrever os casos naturais de abortos e malformações causados pela ingestão de P. pyramidalis em caprinos em municípios do semiárido nordestino.

\section{MATERIAL E MÉTODOS}

As avaliações epidemiológicas, clínicas e as necropsias foram realizadas a partir de visitas a propriedades rurais com histórico de baixa taxa de prenhez, repetição de cios, abortos, malformações e partos distócicos, no município de Pau dos Ferros, Rio Grande do Norte (no período de janeiro de 2014 a janeiro de 2015), e nos municípios de Gurjão e Soledade na Paraíba (no período de janeiro de 2015 a janeiro de 2016). Esses municípios estão localizados no Semiárido, caracterizado por clima seco e muito quente, com apenas duas estações bem definidas, estação chuvosa de fevereiro a maio, e seca o restante do ano (CPRM 2005, IDEMA 2008).

Inicialmente foi feito o mapeamento das propriedades que apresentavam problemas reprodutivos através da aplicação de um questionário (Fig.1). Foram identificadas as propriedades com maior possibilidade de ocorrência de abortos e malformações em fetos e sua relação com o consumo de folhas da árvore Poincianella pyramidalis (Tul.) L.P. Queiroz (=Caesalpinia pyramidalis Tul.). Após a aplicação do questionário foi realizada a avaliação da área onde as cabras tinham acesso. As propriedades que apresentavam infestação de Mimosa tenuiflora e Aspidosperma pyrifolium foram descartadas do estudo, sendo computadas apenas as propriedades com infestação predominante de $P$. pyramidalis.

Foram selecionadas 11 propriedades no município de Gurjão, uma propriedade no município de Soledade e outra em Pau dos Ferros. Os cabritos que nasceram com malformações e permaneceram vivos nas propriedades foram avaliados durante o crescimento e as suas alterações morfológicas foram classificadas.

Nos casos de aborto, os fetos coletados foram submetidos à necropsia e as malformações apresentadas foram classificadas. Amostras de todos os órgãos e da placenta foram colhidas, fixados em formol 10\%, processadas rotineiramente, embebidas em parafina, seccionadas a $5 \mu \mathrm{m}$ e coradas com hematoxilina e eosina para exame histopatológico. Amostras (placenta, fígado, rim, pulmão e baço) foram também colhidas e submetidas a cultivo microbiológico (aeróbio e anaeróbio). 


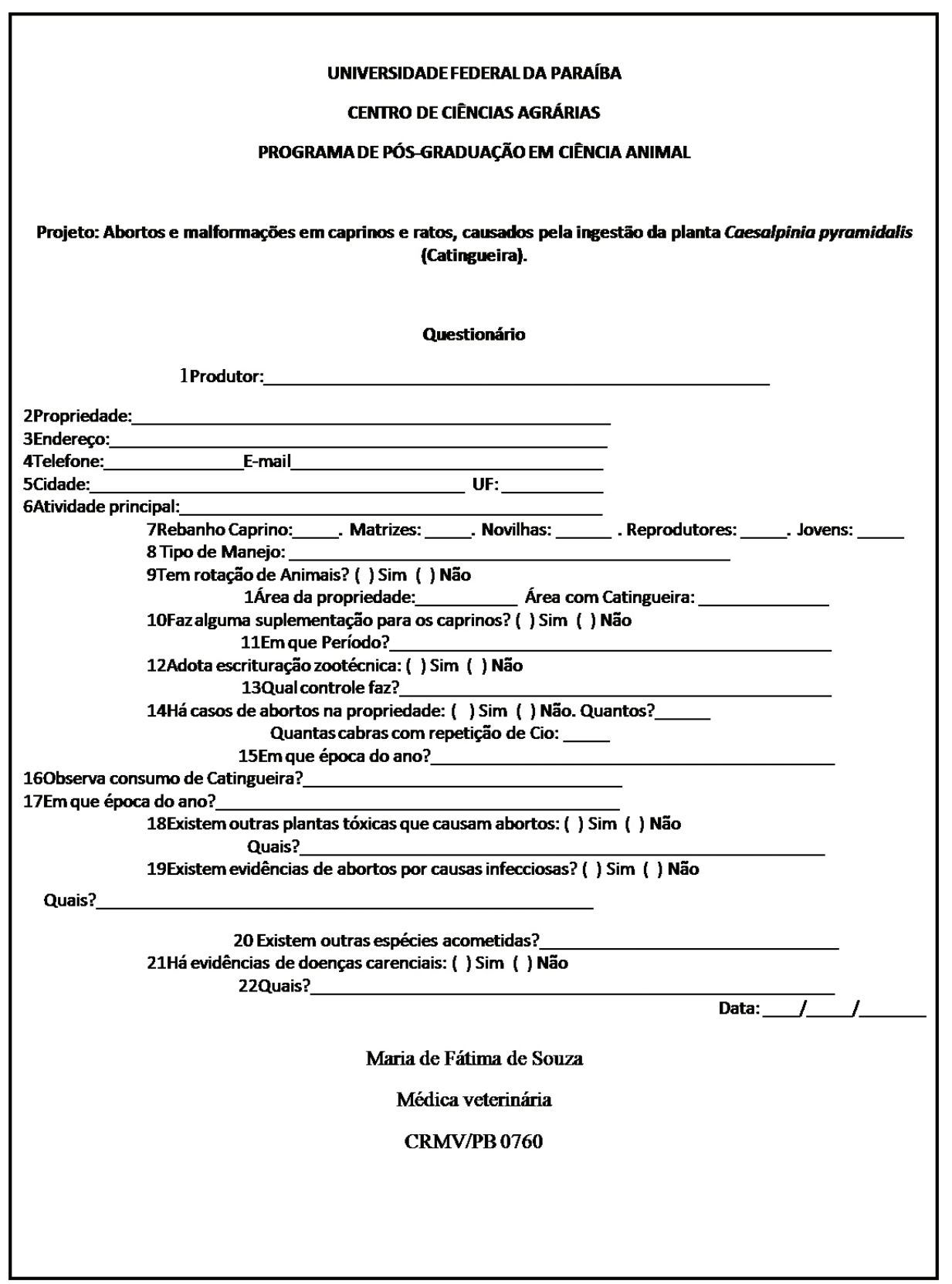

Fig.1. Questionário aplicado aos criadores de caprinos para identificação das propriedades com histórico de malformações associadas ao consumo de plantas tóxicas teratogênicas no Semiárido.

\section{RESULTADOS}

Na primeira propriedade selecionada, localizada no município de Pau dos Ferros, Rio Grande do Norte, foi acometido um rebanho de corte. 0 rebanho era constituído por 80 cabras matrizes mestiças de Boer, três machos Boer puros e 20 cabritos (machos e fêmeas) com um ano de idade. Os caprinos tinham acesso a sal mineral e eram criados extensivamente durante o dia em uma área de cinco hectares e recolhidos à noite para o aprisco.

No final do período de seca (dezembro de 2013 e janeiro de 2014) foi observada a ocorrência de grande número de abortos e o nascimento de cabritos malformados, acometendo $90 \%$ das cabras ( 72 cabras). Os surtos ocorreram nos meses caracterizados por total escassez de pastagens e alguns dias após as chuvas da pré-estação, caracterizadas por baixo volume pluviométrico. Essas chuvas não foram suficientes para germinação e crescimento da pastagem, mas foi suficiente para que a catingueira emitisse brotos (Fig.2), que foram consumidos pelos caprinos. Durante a investigação na propriedade observou-se a presença de grande quantidade de P. pyramidalis com sinais de consumo. Não havia presença de Mimosa tenuiflora e Aspidosperma pyrifolium.

As 36 cabras (45\%) que estavam no início da gestação (até 45 dias) apresentaram morte embrionária e retorno ao cio entre 25-30 dias. As 27 cabras $(33,8 \%)$ que estavam em estágio mais avançado de gestação abortaram fetos 
malformados ou houve a ocorrência de natimortos, associados a partos distócicos, que resultou em morte de quatro cabras. Um total de 15 cabras pariram 15 cabritos vivos, porém, seis cabras pariram seis cabritos que apresentavam múltiplas malformações e outras três cabras pariram cabritos fracos que morreram algumas horas ou poucos dias após o nascimento. Apenas seis cabras pariram seis cabritos que nasceram normais e sobreviveram.
As necropsias de dois fetos machos natimortos, provenientes de gestação não gemelar, acompanhados de suas placentas, revelou artrogripose dos quatro membros (Fig.3), lordose lombar, escoliose cervical em ambos, palatosquise em um feto e queilopalatosquise em outro. A avaliação histopatológica descartou a presença de agentes infecciosos. Não foi observado crescimento de bactérias ou fungos em amostras colhidas dos fetos.
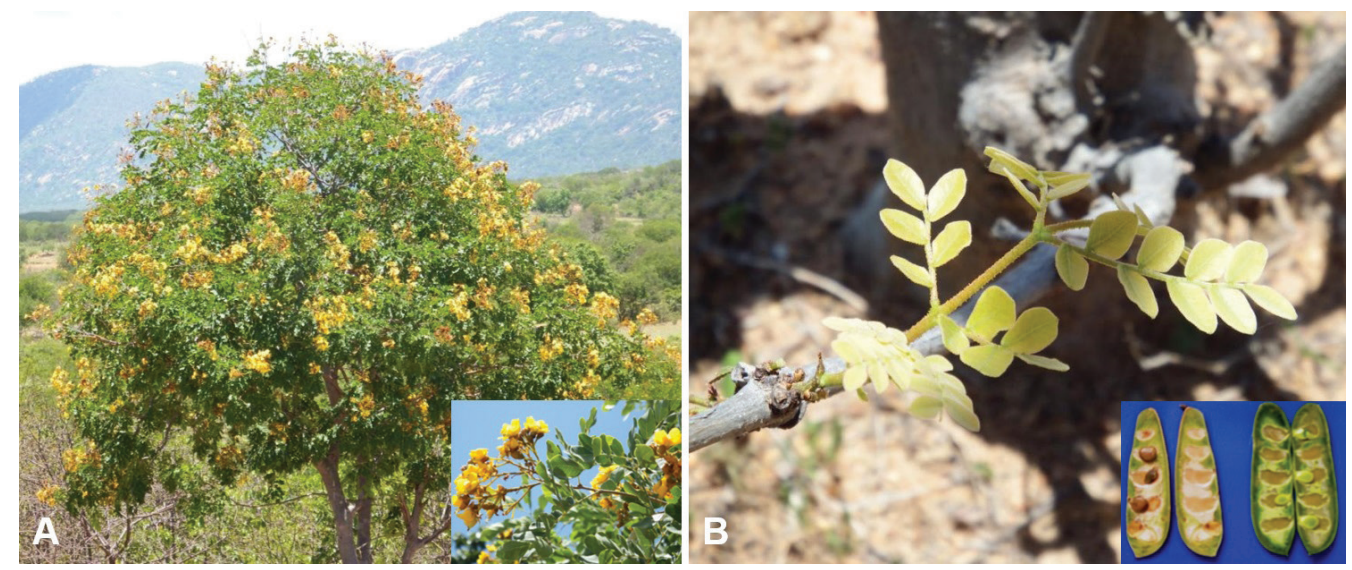

Fig.2. Poincianella pyramidalis (Tul.) L.P. Queiroz (=Caesalpinia pyramidalis Tul.). (A) Planta adulta em fase de floração. No detalhe aproximado notar as flores típicas da planta. (B) Rebrotação da planta após as chuvas da pré-estação. Vagem e sementes no detalhe aproximado.
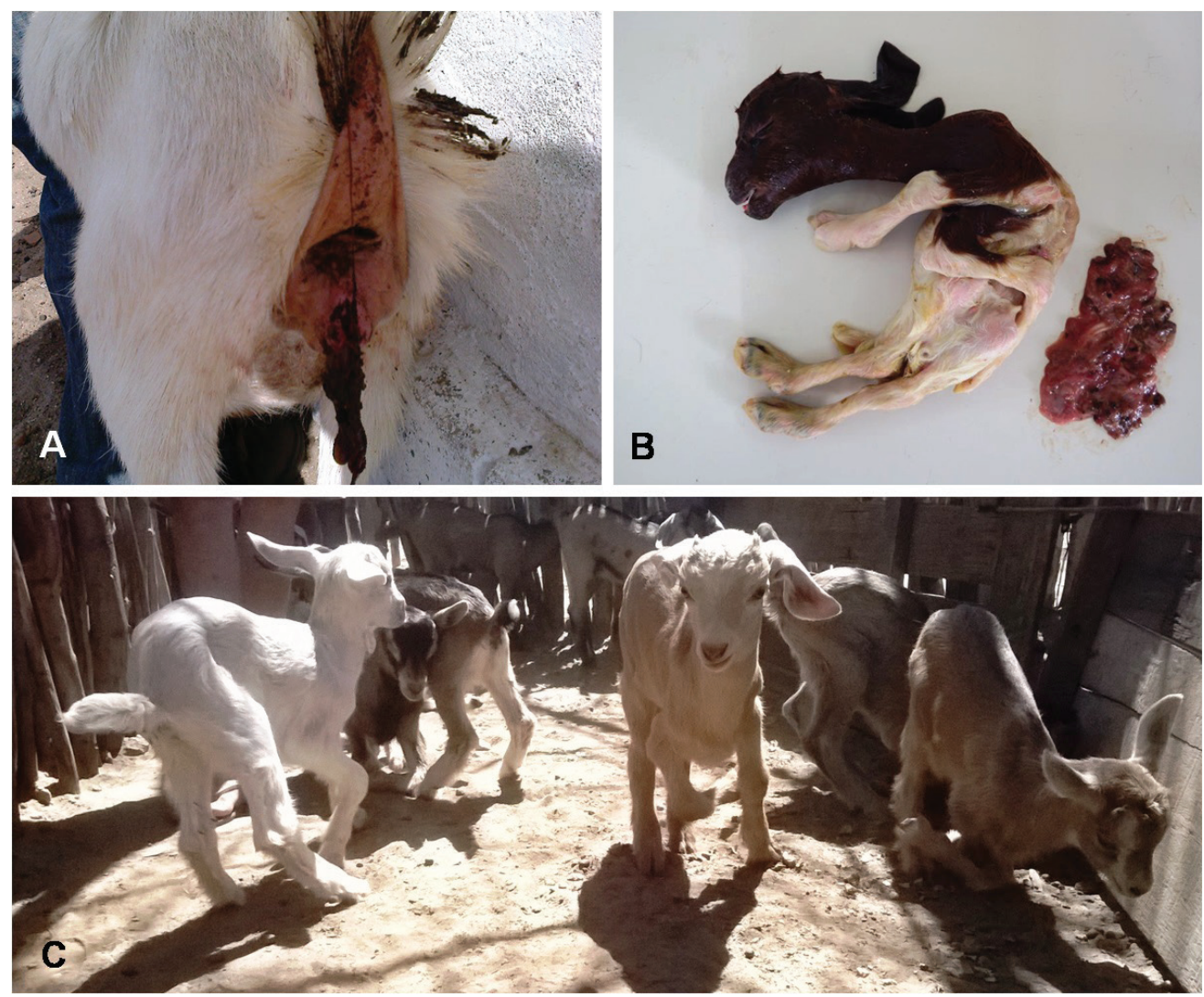

Fig.3. Intoxicação por Poincianella pyramidalis (Tul.) L.P. Queiroz (=Caesalpinia pyramidalis Tul.) em caprinos. (A) Cabra Saanen apresentando retenção de placenta, associada a corrimento vermelho-escuro, quatro dias após abortar um caprino acometido por malformações. (B) Placenta e feto de caprino Boer acometido por artrogripose. Nota-se flexão permanente dos membros torácicos e hipertensão dos membros pélvicos. (C) Rebanho de caprinos mestiços acometidos por artrogripose dos membros torácicos. 
A propriedade foi acompanha por um ano. Durante esse período, as cabras gestantes (desde o momento da cobertura) eram transferidas para outra área da propriedade livre de plantas teratogênicas. Neste período houve apenas um caso de aborto. 0 feto não apresentava anomalias congênitas. A causa do aborto não foi determinada.

Nas 12 propriedades pertencentes aos municípios de Gurjão e Soledade na Paraíba os casos naturais de abortos e malformações acometeram rebanhos de caprinos leiteiros, constituídos por cabras das raças Parda Alpina, Saanen e mestiças dessas raças. As áreas das propriedades que as cabras tinham acesso variaram de 3,5 hectares a 250 hectares, com a média de 95,3 hectares. A maioria das propriedades estudadas adotava o manejo semi-intensivo (oito propriedades), seguido do sistema extensivo (quatro propriedades). No sistema semi-intensivo apenas duas propriedades não suplementavam as cabras, enquanto que no sistema extensivo, metade das propriedades não fornecia suplemento alimentar. Porém, o suplemento fornecido às cabras não era balanceado, consistindo em uma mistura aleatória de grãos (milho, farelo de soja ou torta de algodão), mistura múltipla de sal mineral para caprinos com sal comum e capim elefante ou silagem.

Entre as 12 propriedades, quatro apresentaram casos de malformações em natimortos ou neonatos e em oito propriedades houve histórico de abortos e malformações. Nessas propriedades havia intensa infestação da planta Poincianella pyramidalis. Os casos ocorreram principalmente no final do período da seca, com o início das chuvas da pré-estação chuvosa e rebrotação da "catingueira", ou início do período da seca, quando o pasto estava escasso, mas a planta ainda estava verde.

Em um total de 626 cabras das 12 propriedades foi constado que somente 369 (58,9\%) cabras pariram cabritos normais. Retornos irregulares de cios foram observados em $86(13,7 \%)$, indicando a ocorrência de perda embrionária. Foi computada a ocorrência de 239 casos de abortos ou natimortos geralmente acometidos por malformações, assim como o nascimento de 32 cabritos que apresentavam diferentes malformações e permaneceram vivos. 0 exame clínico revelou que entre esses, 19 (59,3\%) cabritos apresentavam apenas artrogripose dos membros torácicos; cinco $(15,6 \%)$ apenas artrogripose dos membros pélvicos; cinco $(15,6 \%)$ eram casos de artrogripose torácica com escoliose cervical e lombar; três (15,8\%) apresentavam micrognatia; um (5,3\%) cabrito foi acometido por artrogripose torácica com queilosquise; além de um $(5,3 \%)$ caso de queilosquise.

Exames de necropsias e histopatológicos de dois fetos não constataram a presença de agentes infecciosos. Não foi observado crescimento em cultura microbiológica para bactérias ou fungos em amostras colhidas desses fetos ou das placentas.

\section{DISCUSSÃO}

A alta ocorrência de perda embrionária, abortos e malformações, concentradas nas épocas do ano em que a pastagem estava escassa, mas que havia disponibilidade de Poincianella pyramidalis nas propriedades estudadas comprova que esta planta interfere consideravelmente na eficiência reprodutiva dos rebanhos caprinos do Semiárido. Os efeitos tóxicos da catingueira já haviam sidos comprovados em estudo experimental realizado na Bahia com cabras gestantes que consumiram a planta como única fonte forrageira (Reis et al. 2016), mas uma relação direta entre casos naturais de abortos e malformações com o consumo de P. pyramidalis ainda não havia sido investigada. Foi comprovado que os problemas reprodutivos foram controlados na propriedade que adotou manejo correto, mantendo as cabras gestantes em áreas livres de P. pyramidalis.

Os casos de abortos e malformações ocorreram principalmente após o início das primeiras chuvas (chuvas da pré-estação). As chuvas da pré-estação no Semiárido ocorrem entre os meses de novembro e janeiro (Silva et al. 2012). Nesses meses constatou-se que o pasto era escasso, restando como alternativa de alimentação para os caprinos as plantas que apresentavam brotação em decorrência das chuvas da pré-estação, ou seja, predominantemente a árvore $P$. pyramidalis. Casos de aborto e malformações também ocorreram nos rebanhos em que as cabras eram suplementadas, porém, a ração fornecida não era balanceada, provavelmente não atendendo as exigências nutricionais das cabras. A suplementação permitia que a cabras ciclassem e fossem cobertas, mas não impediu que as estas ingerissem folhas da catingueira.

A maioria das propriedades investigadas era pequena, geralmente com alta taxa de lotação, contribuindo para que os caprinos consumissem a planta. Nessas propriedades os criadores tinham o hábito de desmatar as áreas de mata nativa para abrir áreas de pasto, porém as árvores de catingueira não era arrancadas. Na caatinga, os criadores não derrubam a catingueira, pois as folhas da planta que caem ao final do período das chuvas são fonte de alimento para os pequenos ruminantes durante a seca (Queiroz 2009). No presente estudo, constatou-se que os problemas reprodutivos estavam diretamente relacionados, principalmente, ao consumo dos brotos, sem comprovação da associação com o consumo de folhas maduradas que caem ao solo. Estudos experimentais deverão ser realizados com as folhas maduras para comprovação desta observação.

0 percentual de cabras afetadas nos rebanhos foi muito alto, chegando a acometer até $90 \%$ das cabras na propriedade de Pau dos Ferros, na forma de mortalidade embrionária, abortos ou ocorrência de natimortos. Estes dados corroboram com os resultados obtidos na intoxicação experimental por P. pyramidalis em cabras mestiças, realizado na Bahia. Neste estudo experimental, das oito cabras gestantes que ingeriram folhas da planta 75\% (seis cabras) apresentaram problemas reprodutivos ou malformações dos fetos; apenas duas pariram cabritos normais. Os autores verificaram ainda, que $37,5 \%$ das cabras tiveram mortalidade embrionária e reabsorção fetal (Reis et al. 2016).

As lesões observadas nos cabritos malformados, tanto nos que nasceram vivos, quanto nos natimortos, afetaram principalmente os membros torácicos, seguidos dos membros pélvicos, na forma de encurvamento permanente destes membros (artrogripose). Múltiplos fetos abortados apresentavam alterações de coluna, caracterizadas por escoliose, lordose e cifose. Outras alterações acometeram a face e foram caracterizadas por fendas labiais (queilosquise) e palatinas (palatosquise) ou encurtamento mandibular (micrognatia), lesões encontradas também por Reis et al. (2016), nos fetos de cabras que ingeriram $P$. pyramidalis experimentalmente.

Trabalhos anteriores demonstraram que as malformações são uma das principais causas de mortalidade perinatal em pequenos ruminantes no Semiárido (Riet-Correa et al. 2004, Nóbrega Junior et al. 2005), respondendo por $23 \%$ das causas 
de mortes neonatais em cordeiros (Nóbrega Junior et al. 2005) e 7,62\% em cabritos (Medeiros et al. 2005). Está comprovado que a planta conhecida como jurema-preta (Mimosa tenuiflora) é responsável por múltiplas malformações em fetos caprinos no nordeste do Brasil (Dantas et al. 2010). Portanto, é importante verificar a presença de $M$. tenuiflora nas áreas com presença de cabras prenhas, principalmente nos primeiros 60 dias de gestação, considerada a fase mais crítica em relação à ação teratogênica da planta (Dantas et al. 2010). Estas mesmas observações deverão ser indicadas para prevenção de intoxicação por P. pyramidalis, já que as alterações fetais são semelhantes às descritas na intoxicação por $M$. tenuiflora, como fenda palatina, flexão permanente das articulações, braquignatia, escoliose e torcicolo (Pimentel et al. 2007, Dantas et al. 2012).

Em outras partes do mundo, diferentes plantas também causam malformações em caprinos e ovinos. Na América do Norte, plantas como Veratrum californicum, Lupinus spp. e Lathyrus spp., quando ingeridas por pequenos ruminantes prenhas resultam em numerosas malformações fetais, incluindo exencefalia, lisencefalia, ciclopia e ausência de nervos olfatórios (arrinencefalia), fissura palatina, atrogripose, estenose traqueal e morte embrionária (Panter et al. 2013). Porém, essas plantas não são encontradas no semiárido brasileiro.

Entre os agentes teratogênicos virais, o vírus Akabane e o vírus da língua azul podem afetar os fetos de pequenos ruminantes no início da gestação, quando o vírus ultrapassa a barreira transplacentária, resultando em alterações teratogênicas, envolvendo principalmente o sistema nervoso central, que variam desde pequenos cistos até hidranencefalia (Guedes et al. 2016). Infecções em ovelhas e cabras prenhes pelo vírus Akabane pode causar ainda outras malformações que devem ser diferenciadas das malformações induzidas por plantas como a microencefalia e a hidrocefalia (Kurogi et al. 1977, Smith \& Sherman 2009). Nas propriedades do presente estudo, não foram encontrados indícios da ocorrência de língua azul.

Dentre as causas tóxicas de malformações em pequenos ruminantes, as drogas terapêuticas, com níveis de exposição definidas, e em estágios críticos de desenvolvimento fetal podem ser teratogênicas. Os benzimidazois (parabendazol, cambendazol, oxfendazol, netobimina) são teratogênicos para ovinos, causando anormalidade esquelética, renal e vascular, quando administrados entre o $14^{\circ}$ e o $24^{\circ}$ dia de gestação (Radostits et al. 2007). A cortisona, o estradiol, o bismuto, o selênio e as sulfonamidas, podem causar malformações congênitas (Delatour 1983). Nas cabras do presente estudo, não havia histórico da administração de drogas durante a gestação.

\section{CONCLUSÕES}

O consumo de folhas da árvore Poincianella pyramidalis é uma importante causa de malformações e falhas reprodutivas em rebanhos caprinos no Semiárido.

As cabras são acometidas em qualquer fase de gestação, apresentando mortalidade embrionária quando ingerem a catingueira no início da gestação e os fetos geralmente apresentam artrogripose quando a mãe tem acesso á planta no terço final da gestação.
Os problemas ocorrem predominantemente no final do período de seca, após as chuvas da pré-estação ou quando ocorre a rebrotação da árvore.

Os produtores de caprinos devem ser orientados a não permitir que cabras gestantes tenham acesso a P. pyramidalis durante qualquer fase da gestação, principalmente durante os meses da pré-estação chuvosa.

Agradecimentos.- Os autores agradecem aos criadores de caprinos dos municípios de Gurjão, Soledade e Pau dos Ferros que se disponibilizaram a fornecer as informações sobre seus rebanhos e colaboraram com as visitas de campo e avaliação clínica dos caprinos.

\section{REFERÊNCIAS}

Agra M.F., Freitas P.F. \& Barbosa-Filho J.M. 2007. Synopsis of the plants known as medicinal and poisonous in northeast of Brazil. Braz. J. Pharmacogn. 17(1):114-140. http://dx.doi.org/10.1590/S0102-695X2007000100021.

Albuquerque U.P., Medeiros P.M., Almeida A.L.S., Monteiro J.M., Lins Neto E.M.F., Melo J.G. \& Santos J.P. 2007. Medicinal plants of the caatinga (semi-arid) vegetation of Brazil: a quantitative approach. J. Ethnopharmacol. 114(3):325354. http://dx.doi.org/10.1016/j.jep.2007.08.017. PMid:17900836.

Bahia M.V., David J.P. \& David J.M. 2010. Occurrence of biflavones in leaves of Caesalpinea pyramidalis specimens. Quim. Nova 33(6):1297-1300. http:// dx.doi.org/10.1590/S0100-40422010000600015.

CPRM 2005. Diagnóstico do Município de Gado Bravo. Companhia de Pesquisa e Recursos Minerais, Serviço Geológico do Brasil. Disponível em <http://www.cprm.gov.br/publique/Hidrologia/Mapas-e-Publicacoes/ Paraiba---Atlas-Digital-dos-Recursos-Hidricos-Subterraneos-317.html> Acesso em 10 ago. 2015.

Dantas A.F.M., Riet-Correa F., Medeiros R.M.T., Galiza G.J.N., Pimentel L.A., Anjos B.L. \& Mota R.A. 2010. Malformações congênitas em ruminantes no semi-árido do nordeste brasileiro. Pesq. Vet. Bras. 30(10):807-815. http://dx.doi.org/10.1590/S0100-736X2010001000002.

Dantas A.F.M., Riet-Correa F., Medeiros R.M., Lopes J.R., Gardner D.R., Panter K. \& Mota R.A. 2012. Embryonic death in goats caused by the ingestion of Mimosa tenuiflora. Toxicon 59(5):555-557. http://dx.doi.org/10.1016/j. toxicon.2011.11.020. PMid:22178006.

Delatour P. 1983. Some aspects of the teratogenicity of veterinary drugs. Vet. Res. Commun. 7(1/4):125-131. http://dx.doi.org/10.1007/BF02228606. PMid:6665979.

Guedes R.M.C., Brown C.C., Sequeira L.J. \& Reis Junior J.L. 2016. Sistema digestório, p.146-148. In: Santos R.L. \& Alessi A.C. (Eds), Patologia Veterinária. $2^{\text {a }}$ ed. Roca, Rio de Janeiro.

IDEMA 2008. Perfil do seu Município. Instituto de Desenvolvimento Sustentável e Meio Ambiente. Disponível em < http://adcon.rn.gov.br/ACERVO/idema/ DOC/DOC000000000013919.PDF> Acesso em 10 ago. 2015.

Kurogi H., Inaba Y., Takahashi E., Sato K. \& Satoda K. 1977. Kurogi H, Inaba Y, Takahashi E, Sato K, Satoda K.Congenital abnormalities in newborn calves after inoculation of pregnant cows with Akabane vírus. Infect. Immun. 17(2):338-343. PMid:892909.

Luna J.S., Santos A.F., Lima M.R., Omena M.C., Mendonça F.A., Bieber L.W. \& Sant'Ana A.E. 2005. A study of larvicidal and molluscicidal activities of some medicinal plants of northeast brazil. J. Ethnopharmacol. 97(2):199-206. http://dx.doi.org/10.1016/j.jep.2004.10.004. PMid:15707752.

Medeiros J.M., Tabosa I.M., Simões S.V.D., Nóbrega Junior J.E., Vasconcelos J.S. \& Riet-Correa F. 2005. Mortalidade perinatal em caprinos no semiárido da Paraíba. Pesq. Vet. Bras. 25(4):201-206. http://dx.doi.org/10.1590/ S0100-736X2005000400002. 
Medeiros R.M.T., Neto S.A., Riet-Correa F., Schild A.L. \& Sousa N.L. 2004. Mortalidade embrionária e abortos em caprinos causados por Aspidosperma pyrifolium. Pesq. Vet. Bras. 24:42-43.

Melo I.R.B.V., Lages M.C.C., Santos D.P., Maracajá P.B., Rodrigues R.A.P.F. \& Soto-Blanco B. 2013. The pollen of Caesalpinia pyramidalis tul. is toxic to honey bees (apis mellifera). Arthropod-Plant Interactions 7(4):463-466. http://dx.doi.org/10.1007/s11829-013-9254-3.

Mendes C.C., Bahia M.V., David J.M. \& David J.P. 2000. Constituents of Caesalpinea pyramidalis. Fitoterapia 71(2):205-207. http://dx.doi.org/10.1016/S0367326X(99)00145-8. PMid:10727824.

Nóbrega Junior J.E., Riet-Correa F., Nóbrega R.S., Medeiros J.M., Vasconcelos J.S., Simões S.V.D. \& Tabosa I.M. 2005. Mortalidade perinatal de cordeiros no semi-árido da Paraíba. Pesq. Vet. Bras. 25(3):171-178. http://dx.doi. org/10.1590/S0100-736X2005000300008.

Panter K.E., Welch K.D., Gardner D.R. \& Green B.T. 2013. Poisonous plants: effects on embryo and fetal development. Birth Defects Res. 99(4):223-234. http://dx.doi.org/10.1002/bdrc.21053. PMid:24339034.

Pimentel L.A., Riet-Correa F., Gardner D., Panter K.E., Dantas A.F.M., Medeiros R.M.T., Mota R.A. \& Araujo J.A.S. 2007. Mimosa tenuiflora as a cause of malformations in ruminants in the Northeastern Brazilian semiarid rangelands. Vet. Pathol. 44(6):928-931. http://dx.doi.org/10.1354/vp.446-928. PMid:18039908.

Queiroz L.P. 2009. Poincianella pyramidalis (Tul.) L.P. Queiroz, comb. nov., p.180-183. In: Ibid. (Ed), Leguminosas da Caatinga. Universidade Estadual de Feira de Santana, Feira de Santana.

Radostits O.M., Gay C.C., Hinchcliff K.W. \& Constable P.D. 2007. Disease of the newborn, p.127-172. In: Ibid. (Eds), Veterinary Medicine: a textbook of the diseases of cattle, horses, sheep, pigs and goats. 10th ed. Saunders Elsevier, Philadelphia.
Reis S.D.S., Oliveira R.S., Marcelino S.A.C., Macêdo J.T.S.A., Riet-Correa F, Pimentel L.A. \& Pedroso P.M.O. 2016. Congenital malformations and other reproductive losses in goats due to poisoning by Poincianella pyramidalis (Tul.) L.P. Queiroz (=Caesalpinia pyramidalis Tul.). Toxicon 118:91-94. http://dx.doi.org/10.1016/j.toxicon.2016.04.043. PMid:27125469.

Riet-Correa F. \& Medeiros R.M.T. 2001. Intoxicações por plantas em ruminantes no Brasil e no Uruguai: importância econômica, controle e riscos para a saúde pública. Pesq. Vet. Bras. 21(1):38-42. http://dx.doi.org/10.1590/ S0100-736X2001000100008.

Riet-Correa F., Medeiros R.M.T., Neto S.A., Tabosa I.M. \& Pimentel L.A. 2004. Malformações ósseas em caprinos na região semi-árida do nordeste do Brasil. Pesq. Vet. Bras. 24:45-49.

Riet-Correa F., Medeiros R.M.T., Tokarnia C.H. \& Döbereiner J. 2007. Toxic plants for livestock in Brazil: economic impact, toxic species control measures and public heallth implications, p.2-14. In: Panter K.E., Wieremga T.L. \& Pfister J.A. (Eds), Poisonous Plants: global research and solutions. CAB International, Cambridge, USA. http://dx.doi.org/10.1079/978184 5932732.0002 .

Silva L.L., Menezes H.E.A., Dantas R.T., Costa R.F. \& Menezes H.E.A. 2012. Relações das precipitações da pré-estação com o período chuvoso no Estado da Paraíba. Revta Estud. Ambient. 14:44-55.

Silva L.M.M. \& Matos V.P. 1998. Morfologia de frutos, sementes e plântulas de catingueira (Caesalpinea pyramidalis Tul., Caesalpinaceae) e de juazeiro (Zizyphus joazeiro Mart., Rhamnaceae). Revta Bras. Sementes 20(2):263269. http://dx.doi.org/10.17801/0101-3122/rbs.v20n2p263-269.

Smith M.C. \& Sherman D.M. 2009. Goat Medicine. 2nd ed. Wiley-Blackwell, Ames, p.319-338. http://dx.doi.org/10.1002/9780813818825.ch8. 